\title{
CFD COMPUTATION OF THE SAVONIUS ROTOR
}

\author{
Krzysztof Rogowski, RYszard Maroński \\ Warsaw University of Technology, Faculty of Power and Aeronautical Engineering, Warsaw, Poland \\ e-mail:krogowski@meil.pw.edu.pl; maron@meil.pw.edu.pl
}

\begin{abstract}
In this paper, Computational Fluid Dynamics (CFD) analysis of a two-dimensional two-bucket Savonius rotor, using ANSYS Fluent solver, is presented. Computational methods of fluid dynamics are used to estimate the aerodynamic forces acting on the rotor, the torque and the power extracted by the Savonius rotor. In addition, an analysis of the results for different turbulence models is performed. Numerical investigations are compared with the experiment of the Sandia National Laboratories.
\end{abstract}

Keywords: vertical axis wind turbine, simulation, Computational Fluid Dynamics

\section{Introduction}

A wind turbine with the Savonius rotor is usually a vertical axis wind turbine (VAWT). It is a drag-type machine; however, because of an airflow between buckets (the bent sheets of the Savonius rotor), an additional lift force appears on the buckets. The Savonius rotor is the biggest achievement of Finnish inventor Sigurd Johannes Savonius, who patented it in 1927 (Savonius, 1931). The typical Savonius rotor is equipped with two buckets; however, three or more buckets are possible. It usually has an S-shape cross-section. The Savonius-type wind turbine can achieve even $30 \%$ of the maximum power coefficient comparing with $60 \%$ following from Betz theory (Hansen, 2008). However, this turbine needs 30 times greater surface of the buckets as compared with the wing area of a conventional wind turbine to give the same output power. Therefore, the Savonius rotors are small and low energy devices (Szuster, 2000). They can work as water pumps, drives for small electric generators or ventilators (e.g. Flettner Ventilator). Moreover, the Savonius wind turbines can provide water agitation to keep stock ponds ice-free during winter (Paraschivoiu, 2002; Backwell et al., 1977). Anemometers are often Savonius-type turbines due to low costs and reliability. Because of a very low starting torque of the Darrieus-type wind turbine, the Savonius-type wind turbine is sometimes used as a starter for these machines (Alam and Iqbal, 2010; Gupta and Sharma, 2012). Undoubtedly, one of the advantages of the Savonius rotor is the possibility of using it by residents of Third World countries living without electricity. A worn out and empty oil barrel can be used as a low-power device. During rotation of the Savonuius rotor, the Magnus effect appears. This effect depends on the tip speed ratio of the rotor. Although the tip speed ratio of the Savonius rotor is very close to unity and the created lift forces are low, there are investigations of Savonius-type Magnus wind turbines where the Savonius rotor is used to create lift forces for a classical horizontal axis wind turbine (Tokumaru and Dimotakis, 1993; Komatinovic, 2006). The main advantages of the Savonius rotor are as follows: simple and cheap construction of the wind turbine; low noise level; very high starting torque; rotor operation independent of the wind direction. On the other hand, disadvantages of the Savonius-type wind turbine are as follows: lower efficiency in comparison with lift devices; low rotational speed; fluctuations in the torque during operation of the rotor; difficulty in designing high-power wind turbines because of large mass of the rotor. Many shapes and configurations of the Savonius rotor are currently used. Some rotors have the shape of a drill or the two-unit 
two-bucket Savonius rotors rotated $90^{\circ}$ apart. It decreases the pulsation of the torque with the azimuth. Flat circular discs are attached at the ends of the Savonius rotor. Sometimes, an additional shaft which makes the structure more stiff is used (Johnson, 2004; Komatinovic, 2006; Alam and Iqbal, 2010).

In spite of the very simple structure of the Savonius rotor, its aerodynamics is very difficult to analyze. There are not many simplified aerodynamic methods for modeling wind turbines of this type. One model (perhaps the only one) noticed by Paraschivoiu (2002) is a mathematical model proposed by Chauvin, based on the pressure drop on each side of the blades. It enables computing the power of the two-bucket Savonius rotor without any gap between the buckets. However, the Savonius rotor without the gap is only a drag-type device and its maximum power coefficient is much lower than that the one for the rotor with a specific gap width. There are mainly two ways to investigate the aerodynamic performance of the Savonius rotor: computational methods of fluid dynamics and/or via an experiment. In the 70's, experimental research on a large scale of 2 and 3 bucket Savonius configurations were conducted by Blackwell, Sheldahl and Feltz in the Sandia National Laboratories (Blackwell et al., 1977). The aim of that study was to determine torque characteristics of the Savonius rotors. More detailed measurements of the static and dynamic torque were performed by Ushiyama and Nagai in the 80's (Ushiyama and Nagai, 1988). More detailed numerical investigation of the performance of the Savonius wind turbine, based on the discrete vortex method, was performed by Fernando and Modi (1989).

Numerical computations of the flow around the rotor require integration of the Navier-Stokes equations. Direct integration of these equations (Direct Numerical Simulation - DNS) requires very fine mesh and very efficient supercomputers. It gave an impulse for further development of the CFD methods based on time-averaged equations (Reynolds-averaged Navier-Stokes RANS) or spatially averaged equations (Large Eddy Simulation - LES). Using them, large scale problems can be solved and they are satisfactory for many practical applications (ANSYS, 2013).

The Savonius rotor is shown in Fig. 1, wherein the symbols are as follows: $H_{P}$ is the height of the Savonius rotor; $d$ is the diameter; $R$ is the rotor radius; $D_{P}$ is the diameter of the plate; $s$ is the bucket gap width.
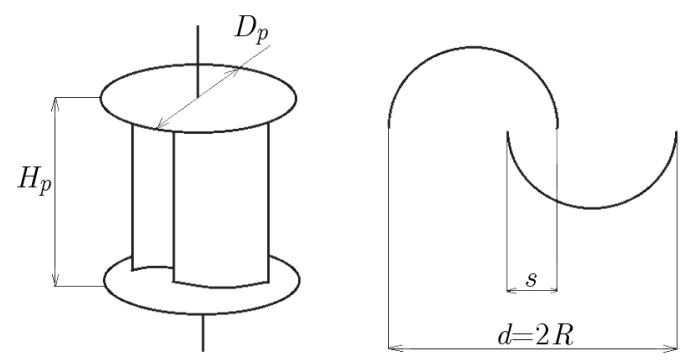

Fig. 1. Savonius rotor

\section{Mathematical formulation}

The computational fluid dynamics (CFD) software, ANSYS Fluent, is used to obtain the power coefficient $C_{P}$ as a function of the tip speed ratio, TSR. The power coefficient $C_{P}$, and the tip speed ratio $T S R$ are as follows

$$
C_{P}=C_{Q} \cdot T S R \quad T S R=\frac{\omega R}{V_{i n}}
$$

where: $\omega$ is the turbine rotational speed; $R$ is the rotor radius of rotation; $V_{i n}$ is the freestream velocity; $C_{Q}$ is the torque coefficient which is given by

$$
C_{Q}=\frac{Q}{\frac{1}{2} \rho V_{i n}^{2} A_{S} R}
$$


where: $\rho$ is the air density; $Q$ is the turbine torque; $A_{S}$ is the rotor swept area which is defined as

$$
A_{S}=(4 r-s) \cdot 1
$$

where: $r$ is the bucket radius; $s$ is the bucket gap width; 1 is a unit height of the Savonius rotor.

Moreover, the side force $F_{y}$, and the downwind force (drag) $F_{x}$, acting on the Savonius rotor as a function of the azimuth position are investigated (see Fig. 2). These forces, expressed as dimensionless coefficients, can be written as

$$
C F_{x}=\frac{F x}{\frac{1}{2} \rho V_{i n}^{2} A_{S}} \quad C F_{y}=\frac{F y}{\frac{1}{2} \rho V_{i n}^{2} A_{S}}
$$

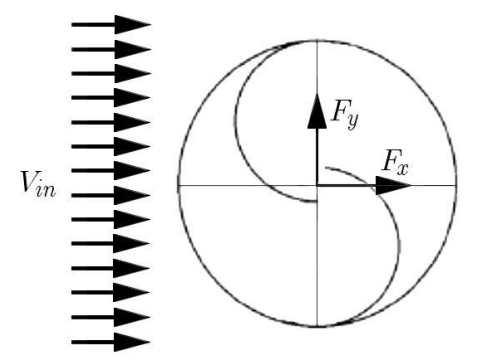

Fig. 2. The side force $F y$ and downwind force $F x$ exerted on the Savonius rotor

\section{Basic fluid flow}

CFD codes use the Reynolds-averaged Navier-Stokes (RANS) equations for computing turbulent flows. The instantaneous continuity and momentum equations can be written as

$$
\begin{aligned}
& \frac{\partial \rho}{\partial t}+\frac{\partial}{\partial x_{i}}\left(\rho u_{i}\right)=0 \\
& \frac{\partial}{\partial t}\left(\rho u_{i}\right)+\frac{\partial}{\partial x_{j}}\left(\rho u_{i} u_{j}\right)=-\frac{\partial p}{\partial x_{i}}+\frac{\partial}{\partial x_{j}}\left[\mu\left(\frac{\partial u_{i}}{\partial x_{j}}+\frac{\partial u_{j}}{\partial x_{i}}-\frac{2}{3} \delta_{i j} \frac{\partial u_{l}}{\partial x_{l}}\right)\right]+\frac{\partial}{\partial x_{j}}\left(-\rho \overline{u_{i}^{\prime} u_{j}^{\prime}}\right)
\end{aligned}
$$

There are many hypotheses which allow one to solve the Reynolds stresses $-\rho \overline{u_{i}^{\prime} u_{j}^{\prime}}$. One of them is the hypothesis of Boussinesq which can be written as

$$
-\rho \overline{u_{i}^{\prime} u_{j}^{\prime}}=\mu_{t}\left(\frac{\partial u_{i}}{\partial x_{j}}+\frac{\partial u_{j}}{\partial x_{i}}\right)-\frac{2}{3}\left(\rho k+\mu_{t} \frac{\partial u_{k}}{\partial x_{k}}\right) \delta_{i j}
$$

where $\mu_{t}$ is the turbulent viscosity.

This hypothesis is commonly used to determine the Reynolds stresses. It is considered in the following turbulence models: the Spalart-Allmaras, the $k-\varepsilon$ and the $k-\omega$. The differences between these models is the way of computing the turbulent viscosity $\mu_{t}$. The Spalart-Allmaras turbulence model solves only one transport equation from which the turbulent viscosity is computed, while the $k-\varepsilon$ and the $k-\omega$ turbulence models introduce two transport equations, for the turbulence kinetic energy $k$, and for its dissipation rate $\varepsilon$ in the case of the $k-\varepsilon$ model, or specific dissipation rate $\omega$ in the case of the $k-\omega$ turbulence model. For the $k-\varepsilon$ and the $k$ - $\omega$ models, the turbulent viscosity $\mu_{t}$ is a function of $k$ and $\varepsilon$ or $k$ and $\omega$. One of the disadvantages of the Boussinesq hypothesis is the constant isotropic scalar, $\mu_{t}$. Therefore, this hypothesis cannot be used for flows with anisotropic turbulence. However, for many flow classes, this hypothesis gives accurate results (Hinze, 1975). 


\subsection{Numerical approach}

The geometrical model of the examined wind turbine (Fig. 3a), used for CFD analysis, consists of two buckets (blades of the Savonius wind turbine). This two-dimensional model of the Savonius rotor is developed based on Blackwell et al. (1977). Usually, the Savonius rotor has upper and lower plates (Fig. 1) so that the energy loss associated with the finite length of the buckets is not large. Therefore, the use of the two-dimensional model is justified. There is the gap $s$ between the buckets of the Savonius rotor (see Fig. 3a). The dimension $s$, one of the main parameters of the Savonius rotor, is defined in the presented study as a distance between the bucket chamber lines. The bucket thickness is $2 \mathrm{~mm}$. The computations presented in this article are performed for the same buckets but for two different lengths of the gap which are expressed as a ratio of the gap length $s$ to the diameter of the bucket $d$. The most important geometrical parameters of the Savonius rotor, which are used during computations, are presented in Table 1.
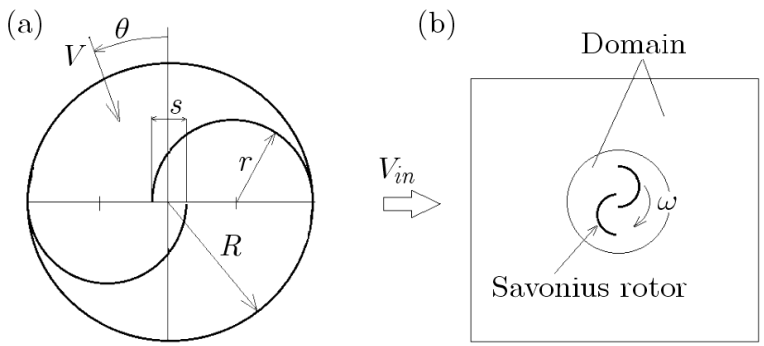

Fig. 3. (a) The basic geometrical parameters of the Savonius wind turbine, (b) the virtual wind tunnel (domain)

Table 1. Basic parameters of the Savonius rotor

\begin{tabular}{|l|c|}
\hline \multicolumn{1}{|c|}{ Parameter } & Value \\
\hline \hline Bucket diameter $d$ & $0.5 \mathrm{~m}$ \\
\hline Gap width ratio $s / d$ & 0.1 and 0.2 \\
\hline Rotor radius $R$ & $0.47 \mathrm{~m}$ and $0.45 \mathrm{~m}$ \\
\hline
\end{tabular}

The problem considered in the work does not involve mesh deformation, therefore the sliding mesh model has been used. This approach is simpler and more efficient in comparison with the dynamic mesh model. The area around the Savonius rotor is modeled as a large square. The rotor is located in the middle of this square area. Moreover, a smaller circle zone around the rotor is created. This circle zone can rotate with the rotor during simulation. The model of the Savonius rotor and the virtual tunnel is presented in Fig. 3b. The area of the virtual wind tunnel has been discretized using triangular elements. An irregular mesh without inflation layers on the blade surfaces is used. The mesh interface is situated between the circle zone and the square zone to allow the exchange of data. There are 41336 cells and 21039 nodes in the presented mesh. Figure 4 presents the mesh used during computations.

\section{Results and discussions}

\subsection{Turbulence models}

There is not any single turbulence model which gives satisfactory results for all classes of flow. The choice of the turbulence model depends on many factors, for example: physics of the flow, accuracy of the solution, computational resources and the amount of time available for simulation. The ANSYS Fluent solver provides many turbulence models. During the authors' 
(a)

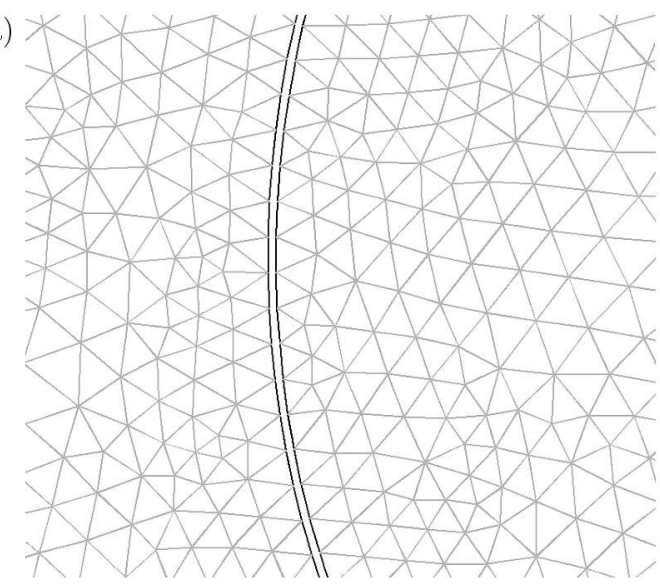

(b)

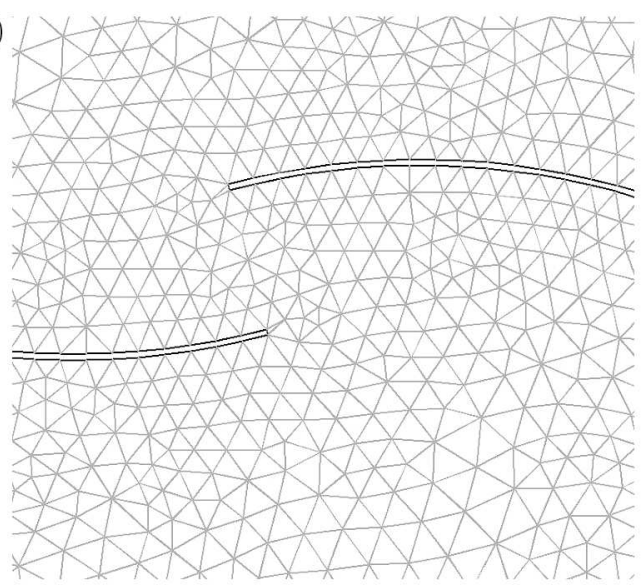

Fig. 4. Example of mesh (the buckets - bold line)

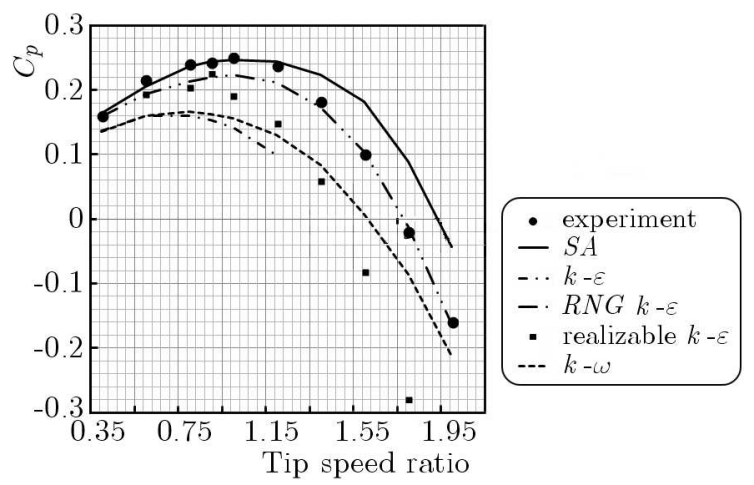

Fig. 5. Power coefficient as a function of the tip speed ratio

investigations, the following turbulence models are used: the SpalartAllmaras $(S A)$, the $k-\varepsilon$, the realizable $k-\varepsilon$, the $R N G k-\varepsilon$ and the $k-\omega$. The CFD results and the experimental results of the power coefficient as a function of the tip speed ratio are presented in Fig. 5. Most of the used turbulence models except for the standard $k-\varepsilon$ and the standard $k-\omega$ models give satisfactory results in the low tip speed ratio range. For a range of tip speed ratios between 0.8 and 2, two turbulence models: the SpalartAllmaras and the $R N G k-\varepsilon$ present results similar to those of the experiment. The results for the SpalartAllmaras turbulence model seem to be overestimated in comparison to the experimental ones. However, the CFD model is two-dimensional and the effects of the finite aspect ratio of the Savonius buckets are neglected. All aerodynamic characteristics of the Savonius rotor, presented in the next Sections, are performed using the SpalartAllmaras turbulence model because it is the simplest model and the accuracy of the solution is satisfactory for the purposes of this work.

\subsection{Dimensions of the virtual wind tunnel}

Appropriate size of the virtual wind tunnel (domain) is very important from the viewpoint of CFD computation. Too small size of the virtual wind tunnel causes too high results of the power coefficients $C_{P}$, whereas too large size causes an increase in computation time. To find an optimal size of the domain, an analysis of the following three dimensions is performed: $W$-width of the virtual wind tunnel, $Z$-length between the inlet of the tunnel and the axis of rotation of the Savonius rotor, and $T$-length between the axis of the Savonius rotor and the outlet (Fig. 6a). 


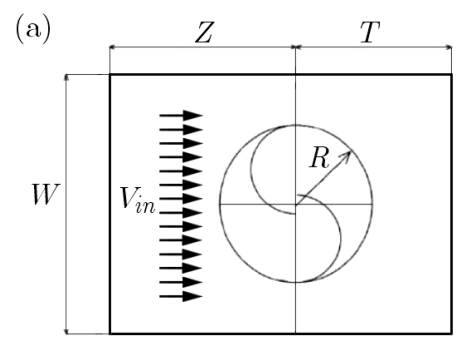

(b)

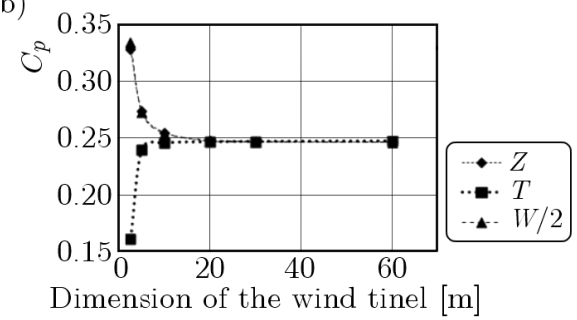

Fig. 6. (a) Dimensions of the domain, (b) the influence of the dimensions on the rotor performance

The results of the power coefficient as a function of length of the domain are presented in Fig. 6b. For the diameter of the rotor of $0.97 \mathrm{~m}$, the dimensions: $W, Z$ and $T$ should be minimum 10 times larger than the rotor diameter. If these lengths are two times larger than the diameter, the power coefficients are overestimated even by $30 \%$.

All CFD results of the Savonius rotor presented in this article are performed for a square domain with the side of $60 \mathrm{~m}$.

\subsection{Power coefficient}

Unsteady flow of the air has been considered in the work. During the computations, aerodynamic forces and the torque are determined at each time step. The time step size corresponds to the azimuth of $0.5 \mathrm{deg}$. The simulation of 4-6 revolutions of the Savonius rotor is required to eliminate the effects associated with initial conditions. Therefore, all simulations presented in this article are performed for 10 revolutions of the rotor. The results of the torque and the forces from the last computed revolution of the rotor are taken for the performance analysis of the Savonius rotor.

Figure 7 presents the torque coefficient $C_{Q}$ as a function of the azimuth position for six values of the tip speed ratio. As it can be observed, the maximum values of the torque coefficient are approximately 0.6 for each tip speed ratio except for the torque coefficient for the tip speed of 0.6. However, the average value of the torque coefficient decreases as the tip speed ratio increases.

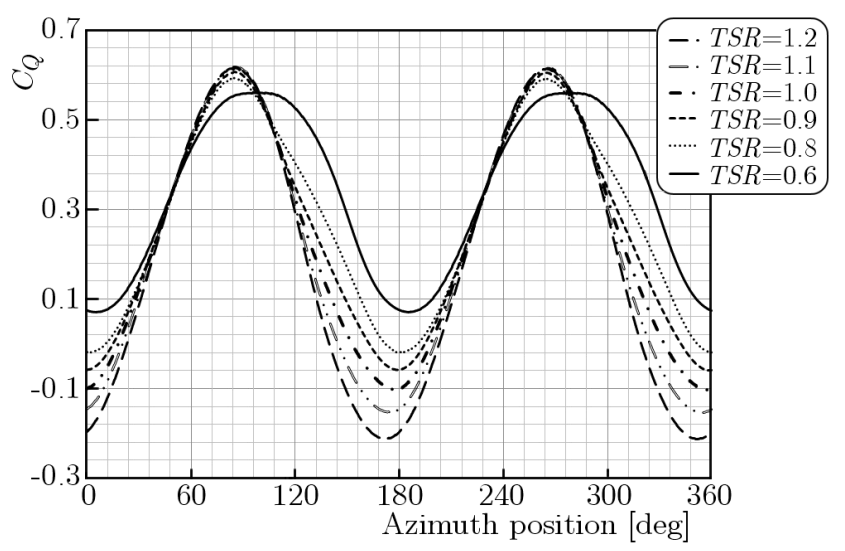

Fig. 7. Torque coefficient as a function of the azimuth position

The mean values of the torque coefficient as a function of the tip speed ratio are presented in Fig. 8a. The obtained results are compared with the experimental torque coefficient. As it can be seen, for the range of the tip speed ratio between 0.4 and 1.2 , the results of the torque coefficient are acceptable. However, the power coefficient $C_{P}$ depends on the tip speed ratio (see Eq. $(2.1)_{1}$ ). Figure $8 \mathrm{~b}$ presents the power coefficient as a function of the tip speed ratio. The maximum power coefficient $C_{P_{\max }}$ of 0.24 is computed for the tip speed ratio of 0.8 . The experimental results are confirmed numerically. 
(a) 0.45

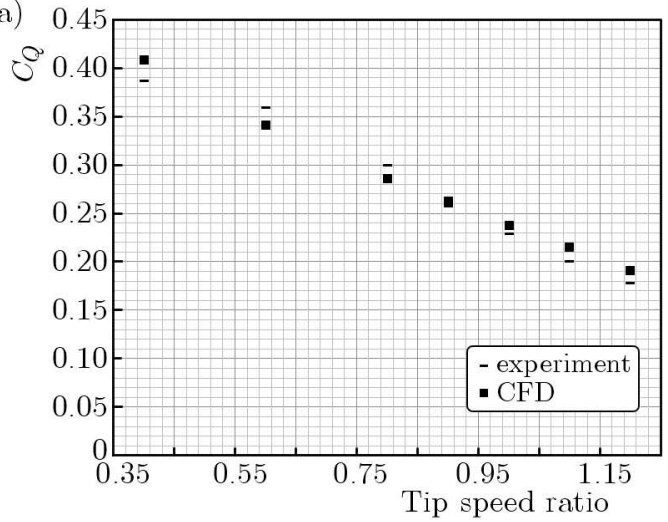

(b)

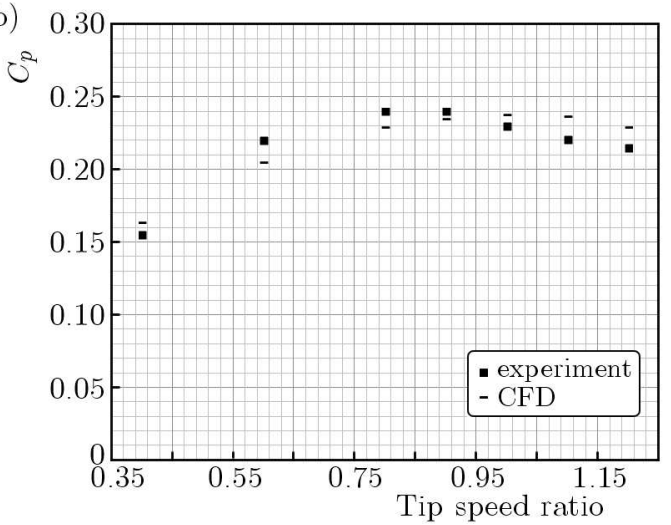

Fig. 8. (a) The torque coefficient as a function of the tip speed ratio, (b) the power coefficient as a function of the tip speed ratio

\subsection{Gap length}

Blackwell et al. (1977) tested different configurations of buckets of the Savonius rotor to find the optimal one. They recommended a configuration consisting of two buckets positioned as it is shown in Fig. 3a, with dimensionless gap width of 0.1-0.15. In this Section, the results of the power coefficient as a function of the tip speed ratio for two dimensionless gap widths are presented. The torque coefficient versus the tip speed ratio is presented in Fig. $9 \mathrm{~b}$ whereas the power coefficient as a function of the tip speed ratio is shown in Fig. 9a. The CFD results of the torque coefficient in comparison with the experimental one seem to be optimistic. Larger differences are observed for the power coefficient. However, it seems to the authors that the computational analysis confirms the results taken from literature. The power coefficient is higher for the dimensionless gap width of 0.1 in comparison with the gap width of 0.2 . This is the most important conclusion for constructors.
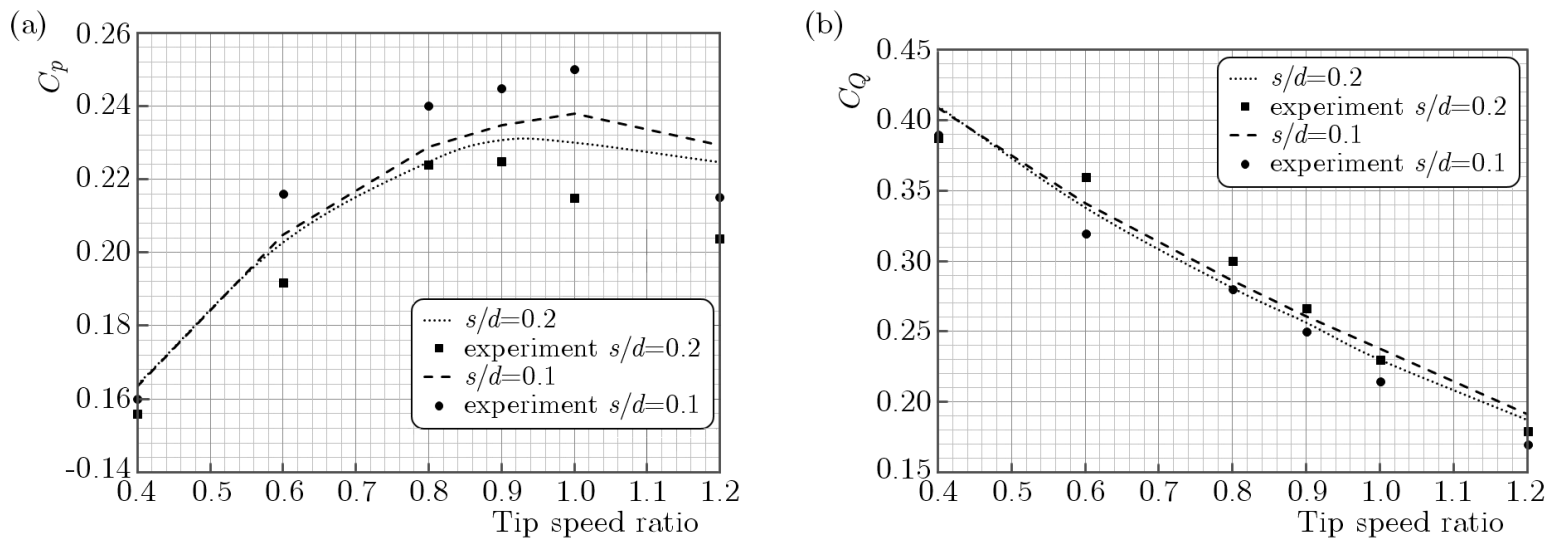

Fig. 9. (a) The torque coefficient as a function of the tip speed ratio, (b) the power coefficient as a function of the tip speed ratio

\subsection{Effect of the Reynolds number}

During experimental tests of Blackwell et al. (1977), it turned out that the power coefficient depends on the Reynolds number. The performance of the Savonius rotor is computed for two velocities of wind, $V_{i n}$ of $7 \mathrm{~m} / \mathrm{s}$ and $14 \mathrm{~m} / \mathrm{s}$. The computed results are shown in Fig. 10. As can be seen, the efficiency of the Savonius rotor is slightly larger for the wind speed of $7 \mathrm{~m} / \mathrm{s}$ in comparison with the results for the wind speed of $14 \mathrm{~m} / \mathrm{s}$. 


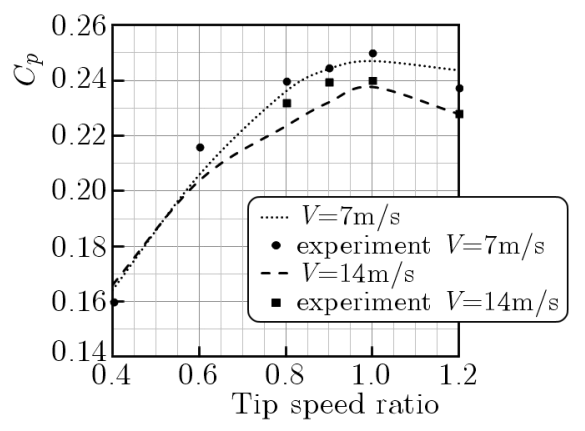

Fig. 10. Power coefficient as a function of the tip speed ratio

\subsection{Influence of the side force}

In this Section, the aerodynamic forces acting on the Savonius rotor as a function of the azimuth position are presented. The reason for which the authors of this article decided to perform these computations is the following sentence from the article by Blackwell et al. (1977): Recent analytical studies, along with unpublished data of the authors, indicate that the Savonius rotor experiences side forces that are of the same order of magnitude as the downwind (drag) force.

(a)

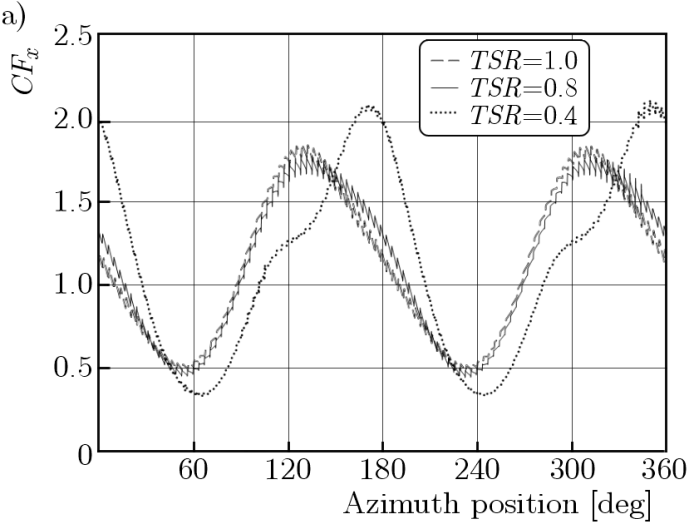

(b)

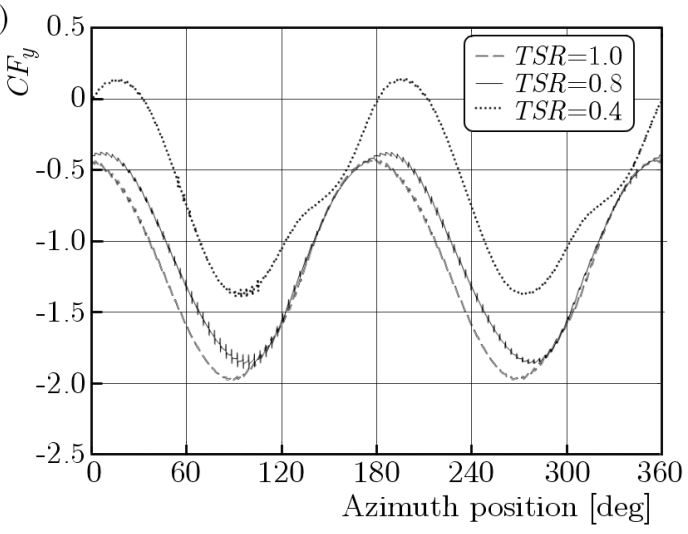

Fig. 11. (a) Drag force coefficient as a function of the azimuth position, (b) the side force coefficient as a function of the azimuth position

Figures $11 \mathrm{a}$ and $11 \mathrm{~b}$ present coefficients of the aerodynamic force: the drag force coefficient $C F_{x}$ and the side force coefficient $C F_{y}$, respectively. Figure 2 shows the vectors of the drag force $F_{x}$ and the side force $F_{y}$. The aerodynamic forces acting on the Savonius rotor vary periodically in time. The results of the drag force coefficient and the side force coefficient for both tip speed ratio of 0.8 and 1.0 differ slightly. For these tip speed ratios $C F_{x}$ is approximately in the range of $0.5-1.8$ whereas $C F_{x}$ is approximately in the range of $-2.0--0.4$. For the tip speed ratio of $0.4 C F_{x}$ varies in the range of $0.35-2.0$ and $C F_{y}$ varies in the range of $-1.4-0.15$. The average values of the force coefficients are shown in Table 2 . As it can be seen, the mean value of $C F_{x}$ is similar for all tip speed ratios while the average value of the side force coefficient increases with an increase in the tip speed ratio. For the tip speed ratio of 0.4 , the mean value of the side force coefficient is about two times lower in comparison with the drag force coefficient, while for the tip speed ratio of 0.8 and 1.0 the side force coefficients are in the same order of magnitude as the drag force coefficients. This conclusion is important from the viewpoint of the design of bearings and dynamics of the shaft. 
Table 2. The average values of the drag force coefficient and the side force coefficient for three tip speed ratios

\begin{tabular}{|c|c|c|}
\hline Tip speed ratio & $C F x$ & $C F y$ \\
\hline \hline 0.4 & 1.1436 & 0.6405 \\
\hline 0.8 & 1.1372 & 1.0824 \\
\hline 1.0 & 1.1383 & 1.1974 \\
\hline
\end{tabular}

\section{Conclusions}

In the present investigation, the aerodynamic efficiency of the Savonius rotor using computational methods of fluid dynamics is studied. The obtained CFD results are compared with the experiment. The conducted analyses have shown that the one-dimensional turbulence model Spalart-Allmaras and mesh without inflation layers can be used for the Savonius rotor applications in the two-dimensional case. The optimum size of the domain has been investigated. This study has demonstrated that the CFD methods confirm the experimental results and can be used to optimize the shape of buckets of the Savonius rotor. According to the results of this research, it can be concluded that the side force acting on the Savonius rotor can be in the same order of magnitude as the drag force, depending on the tip speed ratio.

\section{References}

1. Alam J., Iqbal T., 2010, A low cut-in speed marine current turbine, Journal of Ocean Technology, $\mathbf{5}, 4$

2. ANSYS, 2013, ANSYS Fluent 15.0 Documentation, Fluent Theory Guide

3. Backwell B.F., Sheldahl R.E., Feltz L.V., 1977, Wind Tunnel Performance Data for Twoand Three-Bucket Savonius Rotors, Sandia Laboratories, Report SAND76-0131

4. Fernando M.S.U.K., Modi V.J., 1989, A numerical analysis of the unsteady flow past a Savonius wind turbine, Journal of Wind Engineering and Industrial Aerodynamics, 32, 303-327 item Flettner Ventilator, www.flettner.co.uk

5. Gupta R., Sharma K.K., 2012, Flow physics of a combined Darrieus-Savonius rotor using computational fluid dynamics (CFD), International Research Journal of Engineering Science, Technology and Innovation, 1, 1, 1-13

6. Hansen M.O.L., 2008, Aerodynamics of Wind Turbines, Earthscan, UK, USA

7. Hinze J.O., 1975, Turbulence, McGraw-Hill, New York

8. Johnson G.L., 2004, Wind Energy Systems, www.rpc.com.au, Electronic Edition

9. Komatinovic N., 2006, Investigation of the Savonius-type Magnus Wind Turbine, www.mek.dtu.dk, Master Thesis Project, Technical University of Denmark

10. Paraschivoiu I., 2002, Wind Turbine Design With Emphasis on Darrieus Concept, Presses Internationales Polytechnique

11. Savonius S.J., 1931, The S-rotor and its applications, Mechanical Engineering, 53, 5

12. Szuster T.J., 2000, Wind generators with vertical axis of rotation (in Polish), Ogólnopolskie Forum Odnawialnych Źródeł Energii, Łódź

13. Tokumaru P.T., Dimotakis P.E., 1993, The lift of a cylinder executing rotary motions in a uniform flow, Journal of Fluid Mechanics, 255, 1-10

14. Ushiyama I., NAgai H., 1988, Optimum design configurations and performance of Savonius rotors, Wind Engineering, 12, 1, 59-75 